\title{
Estado del uso de kits de mallas protésicas no reabsorbibles por vía vaginal en cirugía reconstructiva pélvica
}

\begin{abstract}
Antes de comenzar, voy a definir el ámbito de este artículo. Me referiré específicamente al uso de mallas protésicas no reabsorbibles, utilizadas para la reparación del prolapso genital, cuando son usadas por vía vaginal. Se excluyen, por lo tanto, las cintas suburetrales de polipropileno usadas para el tratamiento de la incontinencia urinaria y las mallas protésicas del mismo material utilizadas para reparar el prolapso genital por vía abdominal sin apertura de vagina.
\end{abstract}

Una de cada 10 mujeres será operada por un prolapso genital, correspondiendo un tercio de estas cirugías a reoperaciones (1). El prolapso genital no sólo es prevalente, sino que además, dado que es una condición que en su mayoría se presenta en la postmenopausia, tiene una prevalencia que va en franco aumento. Según el US Census Bureau, en 2050, la población mayor de 65 años será de casi 88 millones de personas, el doble del mismo grupo etario que en $2012(2,3)$.

Hacia mediados de los 80 , las técnicas clásicas por vía vaginal para reparar el descenso de los órganos pélvicos, todas ellas mediante el uso de tejido nativo, no habían podido solucionar el problema, siendo los principales puntos de recurrencia los segmentos apical y anterior, en porcentajes que variaban entre el $30 \%$ y el $70 \%$ (4). Las técnicas eran tantas cómo cirujanos las practicaban. La estandarización para evaluar el grado de descenso de las estructuras, y sobre todo, para evaluar y comparar resultados post operatorios, era precaria. Los conceptos bioestadísticos también estaban en pañales, y lo que se podía encontrar en la literatura eran básicamente series de casos, en el mejor escenario, una cohorte bien seguida. Por otro lado, los conceptos anatómicos sobre soporte pélvico recién se estaban consolidando.

Los años 90 llegaron cargados de novedades, dentro de ellas, el concepto de medicina basada en la evidencia, lo que obligó a mejorar la calidad de los estudios desde el punto de vista epidemiológico. Se estableció además el POP-Q, un sistema de clasificación preciso, reproducible y estandarizable, para evaluar el grado de descenso de los órganos pélvicos y su evolución post operatoria (5), es decir, su grado de éxito en el tiempo. Se pudo iniciar la correcta comparación entre diferentes técnicas, y se definieron los primeros criterios de curación anatómicos para prolapso genital (6). Estos criterios designaban cómo cura o mejoría del prolapso a todo resultado quirúrgico que no se acerque a más de $2 \mathrm{~cm}$ del anillo himeneal. Lo demás era considerado una recidiva. No se consideraron en ese momento los criterios subjetivos de curación.

A principios del $2^{\circ}$ milenio hacen su aparición los Kits de mallas para reparación de prolapso (Prolift $\AA$, Perigee $\AA$, Apogee $\AA$, etc), los cuales mostraron resultados sorprendentes del punto de vista de los criterios de éxito anatómicos para prolapso recién estrenados. La proliferación de Kits de mallas fue importante. En el congreso de IUGA de 2007, en Cancún, México, de 440 presentaciones, $79(18 \%)$ trataban sobre el uso de mallas vía vaginal para prolapso genital (7).

Sin embargo, el éxito y entusiasmo inicial comenzó a disiparse con los años, en la medida que empezaron a comunicarse los resultados de estudios de largo plazo. Las tazas de erosión y dolor post operatorio superaron largamente a las de las técnicas tradicionales, y la resolución de los defectos no era constante en el tiempo. A corto andar comenzaron las demandas, y en 2008 la FDA publicó una advertencia sobre las potenciales complicaciones asociadas al uso de mallas por vía vaginal (8). Estas incluían complicaciones Clavien Dindo 3 y 4 (9), es decir, aquellas lo suficientemente graves cómo para requerir una reoperación, la cual no necesariamente solucionaba el problema, habitualmente dolor intratable.

La FDA creó además una base de datos para recolectar las complicaciones derivadas del uso de estas mallas: MAUDE (Manufacturer and User Device Experience) (10). En 2011, se registraron más de 1500 reportes de complicaciones, de las cuales un $35 \%$ correspondía a erosiones, $31 \%$ a dolor, $17 \%$ a infecciones, y $7 \%$ a dispareunia. En 2011, 
la misma FDA publica una actualización sobre el estado en el uso de las mallas (11), en donde ya no advierte, sino que afirma el hecho de que el uso de mallas de polipropileno por vía vaginal tienen complicaciones severas. En Junio de 2012, mediante una carta dirigida a la FDA, Ethicon/Gynecare anuncia el retiro del mercado norteamericano de sus Kits de Mallas para reparación de prolapso por vía vaginal. Por su parte, Astora Women's Health, anteriormente AMS (American Medical Systems) Women's Health Division, decidió cerrar su operación a nivel global a partir del 31 de Marzo del presente año (https://www.astorahealth.com/).

La pregunta que surge por lo tanto es: ¿cómo es posible que la FDA, un organismo gubernamental americano, estrictísimo en la aprobación de fármacos y dispositivos, no previera la mala evolución de los Kits de mallas para ginecología? La respuesta es muy simple. La FDA nunca aprobó estos dispositivos.

Para que un fármaco llegue a las farmacias, pueden pasar largos años de estudios, desde Fase I a IV, a un altísimo costo. Para las prótesis, la situación es un poco diferente. Estas se clasifican desde las Clases I a la III dependiendo de su complejidad. Las Clase I, las más simples, sólo necesitan unas pocas pruebas de seguridad, o controles generales, para ser aprobadas. Las Clase III corresponden a dispositivos que sustentan la vida, cómo prótesis aórticas y válvulas cardíacas artificiales, o bien dispositivos que potencialmente pueden dañar severamente al paciente. Estas requieren de múltiples estudios de seguridad para su aprobación, proceso conocido cómo Pre Market Approval (PMA). Los dispositivos Clase II, entre los que se encontraban las mallas para prolapso, requieren para su aprobación, además de los controles generales de los Clase I, controles especiales (estudios histológicos, seguimiento, etc.). Sin embargo, estos controles especiales pueden pasarse por alto si se pueden acoger a la tramitación del Pre Market Notification $510(\mathrm{~K})$, el formulario $510(\mathrm{~K})$. Este proceso se conoce cómo Clearance, una "autorización" que se otorga cuando el nuevo dispositivo reúne características que lo hacen "substancialmente equivalente" a un dispositivo predecesor aprobado por la FDA, es decir, tiene la misma intención de uso y las mismas características tecnológicas que su predecesor, ó tiene la misma intención de uso, pero con diferentes características tecnológicas, a lo cual se agrega información nueva. En otras palabras, no es necesario demostrar científicamente que es al menos tan seguro y efectivo como su predecesor. Ahora, este dispositivo anterior o predecesor, también puede haber sido aprobado por medio del formulario $510(\mathrm{~K})$.

Es de hecho posible seguir el rastro de las autorizaciones mediante el Formulario 510 (K) en la página de la FDA (http://www.fda.gov/MedicalDevices/ default. htm).La secuencia es la siguiente:

En 1996, Boston Scientific lanzó al mercado un producto llamado ProteGen®, una malla con cubierta de colágeno que se anclaba al hueso púbico para el tratamiento de la incontinencia de orina de esfuerzo. No necesitó controles especiales, pues se usó el formulario $510(\mathrm{~K})$, y se basó en el uso de

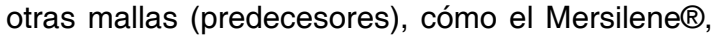
Marlex®, Gore-Tex® y el Trelex®. En 1999, debido a una tasa de erosiones del $50 \%$, un $17 \%$ de fístulas uretrovaginales y a una menor efectividad que las técnicas tradicionales, además de una cantidad importante de juicios en curso, fue retirada del mercado. En 1998, sin embargo, Ethicon/Gynecare, usando el formulario $510(\mathrm{~K})$ y a ProteGen® cómo su predecesor, logró que TVT® fuera autorizado para ser vendido. En Marzo de 2001, Tyco introdujo el Tunelizador I.V.S®, usando el formulario 510 (K) y a TVT cómo su predecesor. En 2002, Ethicon/ Gynecare logró por la misma vía la autorización de la malla Gynemesh® PS. En 2003, Apogee®, de American Medical Systems, logró la autorización usando cómo predecesor al Tunelizador I.V.S.®. Finalmente, en 2008, usando cómo predecesores a Apogee $₫$, Perigee $₫$ y Gynemesh $₫$, es autorizado, siempre mediante el formulario $510(\mathrm{~K})$, el uso de Prolift $®$ y Profilt-M®.

En ninguna de estas autorizaciones se exigió evidencia científica alguna, sólo papeles que demostraran que eran lo suficientemente parecidos al producto predecesor, independiente de si el predecesor había sido retirado del mercado por sus desastrosos resultados, cómo es el caso con ProteGen®. O sea, la aprobación de los kits de mallas más usados a nivel mundial se realizó sin ninguna evidencia científica mediante, basados en un dispositivo que duró 3 años en el mercado, retirado por tasas de complicaciones impresentables.

¿Debería entonces proscribirse el uso de estos dispositivos para el futuro? El consenso de muchas sociedades científicas e importantes centros de referencia $(12,13,14,15,16,17)$ es que no, dado que hay grupos de pacientes que podrían aún beneficiarse de ellas, por ejemplo, pacientes con recidiva de prolapso después de una primera cirugía, pacientes con alto riesgo de recidiva en su cirugía primaria y/o con condiciones que hagan menos deseable un abordaje abdominal. Se hace mandatorio, eso sí, consensuar estos procedimientos con los pacientes, explicando sus posibles complicaciones, su tasa de ocurrencia, y los métodos para repararlas. Además, después de varios años de uso, conocemos en forma más acabada el perfil de seguridad de estos dispositivos y sus resultados, con lo cual podemos evaluar con una base más sólida, y, en conjunto con la paciente los riesgos y beneficios de utilizarlos en cada caso. 
Sin embargo, también existe amplio consenso en que su uso debe ser limitado, en manos de cirujanos experimentados, e idealmente en centros de referencia, para que los resultados se acerquen lo más posible al óptimo.

Probablemente la lección más importante que nos debe quedar del uso de mallas por vía transvaginal para la corrección del prolapso genital, es que antes de implementar cualquier tratamiento, sea este médico o quirúrgico, debemos revisar la bibliografía que apoye su uso. Si esta es escasa o inexistente, no es necesario desecharlo, pero se debe enfocar cómo un tratamiento experimental, para de esa forma poder ofrecérselo a nuestras pacientes en la forma correcta, hasta que la evidencia muestre que es un procedimiento razonablemente seguro y eficaz. Cómo dijo Confucio: "Aprender sin pensar es trabajo perdido, pensar sin aprender es peligroso"

\section{Dr. Carlos Wenzel Dumay Presidente, Sociedad de Patología de Piso Pélvico de Chile. \\ Unidad de Patología de Piso Pélvico, Hospital Clínico Universidad de Chile. Unidad de Patología de Piso Pélvico, Clínica Alemana de Santiago.}

\section{REFERENCIAS}

1. Olsen AL, Smith VJ, Bergstrom JO, Colling JC, Clark $A L$. Epidemiology of surgically managed pelvic organ prolapse and urinary incontinence. Obstet Gynecol 1997;89(4):501-6.

2. Ortman JM, Velkoff VA, Hogan $\mathrm{H}$, et al. An aging nation: the older population in the United States. Washington DC US Census Bur 2014;25-1140.

3. Smith FJ, Holman CDJ, Moorin RE, Tsokos N. Lifetime risk of undergoing surgery for pelvic organ prolapse. Obstet Gynecol 2010;116(5):1096-100.

4. Swift SE. "First do no harm" and the emerging story of the vaginal reconstructive mesh implant. Int Urogynecology J 2007;18(9):983-4.

5. Bump RC, Mattiasson A, Bø K, Brubaker LP, DeLancey JO, Klarskov $P$, et al. The standardization of terminology of female pelvic organ prolapse and pelvic floor dysfunction. Am J Obstet Gynecol 1996;175(1):10-7.

6. Weber AM, Abrams P, Brubaker L, Cundiff G, Davis $\mathrm{G}$, Dmochowski RR, et al. The Standardization of
Terminology for Researchers in Female Pelvic Floor Disorders. Int Urogynecology J 2001;12(3):178-86.

7. 32nd Annual IUGA Meeting, Cancún, Mexico, 12--16 June 2007. Int Urogynecol J 2007;18(1). Disponible en: http://link.springer.com/journal/192/18/1/suppl/page/1.

8. Public Health Notifications (Medical Devices) - FDA Public Health Notification: Serious Complications Associated with Transvaginal Placement of Surgical Mesh in Repair of Pelvic Organ Prolapse and Stress Urinary Incontinence. Disponible en: http://www.fda. gov/MedicalDevices/Safety/AlertsandNotices/PublicHealthNotifications/ucm061976.htm.

9. Clavien PA, Barkun J, de Oliveira ML, Vauthey JN, Dindo D, Schulick RD, et al. The Clavien-Dindo classification of surgical complications: five-year experience. Ann Surg 2009;250(2):187-96.

10. FDA. Surgical Mesh for POP and SUI Repair FDA Executive Summary. agosto de 2011. Disponible en: http://citeseerx.ist.psu.edu/viewdoc/download?doi=10 1.1.666.6831\&rep=rep $1 \&$ type $=$ pdf.

11. UPDATE on Serious Complications Associated with Transvaginal Placement of Surgical Mesh for Pelvic Organ Prolapse: FDA Safety Communication. Disponible en: http://www.fda.gov/MedicalDevices/Safety/ AlertsandNotices/ucm262435.htm

12. AUA Position Statement on the Use of Vaginal Mesh for the Repair of Pelvic Organ Prolapse: American Urological Association. Disponible en: https://www. auanet.org/education/vaginal-mesh-for-pelvic-organprolapse.cfm

13. NHS England » Mesh working group. Disponible en: https://www.england.nhs.uk/ourwork/qual-clin-lead/ mesh/

14. Transvaginal mesh complications: Get the facts Mayo Clinic Disponible en: http://www.mayoclinic.org/ diseases-conditions/pelvic-organ-prolapse/in-depth/ transvaginal-mesh-complications/art-20110300? $p=1$

15. Bjelic-Radisic V, Aigmueller T, Preyer O, Ralph G, Geiss I, Müller G, et al. Vaginal prolapse surgery with transvaginal mesh: results of the Austrian registry. Int Urogynecology J 2014;25(8):1047-52.

16. Vaginal Placement of Synthetic Mesh for Pelvic Organ Prolapse - ACOG. Disponible en: http://www. acog.org/Resources-And-Publications/CommitteeOpinions/Committee-on-Gynecologic-Practice/Vaginal-Placement-of-Synthetic-Mesh-for-Pelvic-OrganProlapse.

17. Walter JE, Lovatsis D, Easton W, Epp A, Farrell SA, Girouard $L$, et al. Transvaginal mesh procedures for pelvic organ prolapse. J Obstet Gynaecol Can 2011;33(2):168-74. 